\title{
DIRECT AND INTERACTIVE EFFECTS OF ENEMIES AND MUTUALISTS ON PLANT PERFORMANCE: A META-ANALYSIS
}

\author{
William F. Morris, ${ }^{1,12}$ Ruth A. Hufbauer, ${ }^{2}$ Anurag A. Agrawal, ${ }^{3}$ James D. Bever,${ }^{4}$ Victoria A. Borowicz, ${ }^{5}$ \\ Gregory S. Gilbert, ${ }^{6}$ John L. Maron, ${ }^{7}$ Charles E. Mitchell, ${ }^{8}$ Ingrid M. Parker, ${ }^{9}$ Alison G. Power, ${ }^{3}$ \\ Mark E. Torchin, ${ }^{10}$ and Diego P. VÁzquez ${ }^{11}$ \\ ${ }^{1}$ Department of Biology, Duke University, Box 90338, Durham, North Carolina 27708-0338 USA \\ ${ }^{2}$ Department of Bioagricultural Sciences and Pest Management, Colorado State University, Fort Collins, Colorado 80523-1177 USA \\ ${ }^{3}$ Department of Ecology and Evolutionary Biology, Cornell University, Ithaca, New York 14853 USA \\ ${ }^{4}$ Department of Biology, Indiana University, Bloomington, Indiana 47405 USA \\ ${ }^{5}$ Behavior, Ecology, Evolution, and Systematics Section, Department of Biological Sciences, Illinois State University, \\ Normal, Ilinois 61790-4120 USA \\ ${ }^{6}$ Department of Environmental Studies, 1156 High Street, University of California, Santa Cruz, California 95064 USA \\ ${ }^{7}$ Division of Biological Sciences, University of Montana, Missoula, Montana 59812 USA \\ ${ }^{8}$ Department of Biology and Curriculum in Ecology, University of North Carolina, Chapel Hill, North Carolina 27599-3280 USA \\ ${ }^{9}$ Department of Ecology and Evolutionary Biology, University of California, Santa Cruz, California 95064 USA \\ ${ }^{10}$ Smithsonian Tropical Research Institute, Apartado 0843-03092, Balboa, Ancon, Republic of Panama \\ ${ }^{11}$ Instituto Argentino de Investigaciones de las Zonas Áridas, Centro Regional de Investigaciones Científicas y Tecnológicas, \\ Av. Ruiz Leal s/n, (5500) Mendoza, Argentina
}

\begin{abstract}
Plants engage in multiple, simultaneous interactions with other species; some (enemies) reduce and others (mutualists) enhance plant performance. Moreover, effects of different species may not be independent of one another; for example, enemies may compete, reducing their negative impact on a plant. The magnitudes of positive and negative effects, as well as the frequency of interactive effects and whether they tend to enhance or depress plant performance, have never been comprehensively assessed across the many published studies on plant-enemy and plant-mutualist interactions. We performed a meta-analysis of experiments in which two enemies, two mutualists, or an enemy and a mutualist were manipulated factorially. Specifically, we performed a factorial meta-analysis using the log response ratio. We found that the magnitude of (negative) enemy effects was greater than that of (positive) mutualist effects in isolation, but in the presence of other species, the two effects were of comparable magnitude. Hence studies evaluating single-species effects of mutualists may underestimate the true effects found in natural settings, where multiple interactions are the norm and indirect effects are possible. Enemies did not on average influence the effects on plant performance of other enemies, nor did mutualists influence the effects of mutualists. However, these averages mask significant and large, but positive or negative, interactions in individual studies. In contrast, mutualists ameliorated the negative effects of enemies in a manner that benefited plants; this overall effect was driven by interactions between pathogens and belowground mutualists (bacteria and mycorrhizal fungi). The high frequency of significant interactive effects suggests a widespread potential for diffuse rather than pairwise coevolutionary interactions between plants and their enemies and mutualists. Pollinators and mycorrhizal fungi enhanced plant performance more than did bacterial mutualists. In the greenhouse (but not the field), pathogens reduced plant performance more than did herbivores, pathogens were more damaging to herbaceous than to woody plants, and herbivores were more damaging to crop than to non-crop plants (suggesting evolutionary change in plants or herbivores following crop domestication). We discuss how observed differences in effect size might be confounded with methodological differences among studies.

Key words: factorial experiment; Hedges' $d$; herbivore; interaction effect; log response ratio; metaanalysis; mutualist; natural enemy; pathogen; plant performance.
\end{abstract}

\section{INTRODUCTION}

Plants engage in multiple biotic interactions that affect their survival, growth, and reproduction and

Manuscript received 14 March 2006; revised 14 July 2006; accepted 18 July 2006; final version received 18 September 2006. Corresponding Editor: J. T. Cronin.

12 E-mail: wfmorris@duke.edu consequently influence the primary productivity of natural ecosystems, agricultural yield, the invasion success of exotic plants, and the evolution of plant traits such as defenses and rewards. Interactions with competitors, herbivores, pathogens, and nectar robbers typically reduce plant performance, while interactions with facilitators, pollinators, seed dispersers, defenders, and fungi and bacteria that mediate nutrient acquisition 
typically enhance it. Yet how the overall magnitudes of such negative and positive biotic interactions compare is at present unknown. Moreover, plants interact with multiple organisms simultaneously. While it is well known that engaging in one biotic interaction has the potential to alter the effects of other interactions, we know little about how frequently such interactive effects occur or whether the net effects tend to be more beneficial or more detrimental to the plant than would be expected from an independent effects model. Interactive effects have pervasive implications. In community ecology, the existence of interactive effects implies that community dynamics cannot be predicted by the interaction coefficients estimated in pairwise experiments (Wilbur 1972, Neill 1974, Wootton 1993), and in evolutionary ecology, interactive effects may cause the selective impact that one species imposes on plants to vary with community context (Hougen-Eitzman and Rausher 1994). In biological control of invasive weeds, the potential for antagonistic interactions between biocontrol agents has underlain the argument for limiting the number of species introduced (McEvoy and Coombs 1999, Denoth et al. 2002), while the opposing argument, that multiple agents may have synergistic effects if the stress imposed by one agent renders the plant even more susceptible to another, is also plausible. Thus understanding the general magnitudes of the direct and interactive effects on plants of different types of biotic interactions is of fundamental basic and applied importance.

Many individual studies have examined how different biotic interactions, both singly and in combination, influence plant performance. Meta-analysis (Gurevitch and Hedges 2001) provides a useful tool for extracting general results from a suite of individual studies. Previous meta-analyses have gauged the negative effects of competitors (Gurevitch et al. 1992, Goldberg et al. 1999, Maestre et al. 2005), herbivores (Bigger and Marvier 1998, Hawkes and Sullivan 2001, Yeo 2005), pathogens (Rosenberg et al. 2004), and nectar robbers (Irwin et al. 2001). In contrast, meta-analyses evaluating interactions that positively affect individual plant performance have focused only on mycorrhizal mutualisms (Borowicz 2001) and plant-plant facilitation (Goldberg et al. 1999, Gomez-Aparicio et al. 2004, Maestre et al. 2005 [also see Lortie and Callaway 2006 and Maestre et al. 2006]). No meta-analyses have compared the effects of different types of mutualists. Similarly, only two meta-analyses have examined whether one biotic interaction influences the magnitude of another. Gurevitch et al. (2000) asked whether herbivory significantly influenced the effect of competition across a set of studies that reported factorial manipulations of herbivores and competitors (also see Hamback and Beckerman [2003]), and Borowicz (2001) analyzed whether arbuscular mycorrhizal fungi influenced the effects of fungal pathogens and nematodes. No meta-analysis has examined more generally whether one natural enemy influences the effect of another, nor tested broadly for nonindependent effects of enemies and mutualists.

Here, we report the results of a meta-analysis of 36 enemy-enemy, 10 mutualist-mutualist, and 114 enemymutualist factorial experiments. These studies span a range of plant life histories, natural enemy and mutualist types, environments, and response variables used to quantify plant performance. We focus our meta-analysis on four questions that have not been addressed in previous meta-analyses. First, do the magnitudes of the effects of enemies and mutualists on plant performance differ on average? Second, do different types of natural enemies (notably herbivores and pathogens) or different types of mutualists (notably pollinators, mycorrhizal fungi, and mutualistic bacteria) differ in the size of their effects on plant performance? Third, if two enemies, two mutualists, or an enemy and a mutualist co-occur, then on average is the net effect on the plant less than, equal to, or greater than the sum of the separate effects of the two species? Fourth, do environmental conditions (i.e., field vs. greenhouse) and plant characteristics (herbaceous vs. woody, crop vs. non-crop) influence the direct and interactive effects of enemies and mutualists? We perform a factorial meta-analysis using the log response ratio, $L$, as a measure of effect size (Hedges et al. 1999). We justify the use of $L$ in Methods: Calculation and comparison of effect sizes.

\section{Methods}

\section{Compilation of the data set}

Our meta-analysis included all studies we found that performed a fully factorial manipulation of two enemies, two mutualists, or an enemy and a mutualist and that reported data on individual plant performance, measures of variation among replicate plants within treatments, and sample sizes (Appendix A). We identified appropriate studies through Web of Science searches, from our own knowledge of the literature, and by checking references in review articles (e.g., Strauss and Irwin 2004) and published meta-analyses (e.g., Borowicz 2001). Henceforth, we use "agents" to refer to species (whether enemies or mutualists) interacting with plants. Many of the studies manipulated two species of agents, but some manipulated entire guilds (e.g., aboveground vs. belowground herbivores). We also incorporated a few studies that used artificial herbivory (e.g., clipping with scissors) when the authors argued that it mimicked natural herbivore effects. We used both field and greenhouse experiments and included both herbaceous and woody and both crop and non-crop species. Most studies were addition experiments, but we included five removal and 10 mixed addition/removal experiments. We refer to the treatment in which both agents were absent as the "control." The original studies quantified agent effects by measuring plant size (e.g., biomass, stem height; 121 studies), reproductive output (e.g., seed set, seedling recruitment; 
36 studies), survival (two studies), or population growth rate (one study). When papers measured performance repeatedly, we used only the final measurements. If the entire factorial design was crossed with additional treatments, such as ambient and increased $\mathrm{CO}_{2}$, we included only the ambient treatment.

Some papers report on multiple pairs of agents manipulated factorially using a common control. In such cases, the effect sizes for different agent pairs are not statistically independent; however, using only a single agent pair decreases the number of different species pairs in the analysis. Some papers repeated experiments with the same pair of agents on the same plant species multiple times (e.g., in different fields or years), each with an independent control. While these replicate experiments are statistically independent, including all of them could bias our results through overrepresentation of some species combinations. We analyzed the full data set, including experiments sharing controls and experiments repeated with the same agent pair, but we also sampled to form three reduced data sets. In one, we used only those studies from a single paper that used different agents even if they shared a control. In the second, we included data only if the controls were independent, even if the same agent pair was repeated. In the third, most conservative approach, we included data that used different agents and independent controls. In subsampling, a single study was randomly chosen from each set of nonindependent studies, effect sizes were computed as described below, and the process was repeated 5000 times with replacement.

\section{Calculation and comparison of effect sizes}

Most ecological meta-analyses have measured effect sizes using Hedges' $d$ (Hedges and Olkin 1985) which, in the present context, is the difference in mean plant performance when an interacting species is present vs. absent divided by the pooled standard deviation within treatments. However, because $d$ is measured in standard deviation units, a small absolute difference in mean performance can yield a large effect size if the variance in performance within treatments is low. Moreover, two studies can have the same effect size even if the difference in mean performance is small in one study but large in the other (i.e., if the first also has lower within-treatment variability). Frequently we are interested in the actual difference in mean performance. For example, for gauging effects on primary productivity, we would want to know by what proportion herbivores reduce plant biomass on average, and to gauge the magnitude of selection exerted by pollinators, we would want to know the proportional increase in seed production. Consequently, we measured effect sizes using the response ratio, which is the ratio of mean plant performance in the presence vs. absence of an interacting species. For example, a response ratio of 0.8 indicates that the interacting species (an enemy) reduced plant performance by $20 \%$ on average, while a ratio of 1.2 indicates the interacting species is a mutualist that increased mean performance by $20 \%$. Additionally, the response ratio assumes effects of different agents are multiplicative, which may be more realistic biologically (Sih et al. 1998), while $d$ assumes additive effects. To conduct our factorial meta-analysis, we extended the approach of Gurevitch et al. (2000), which uses Hedges' $d$, to the response ratio (also see Hawkes and Sullivan [2001]), but because we compare our results to past meta-analyses, we also report our results using Hedges' $d$ in Appendix C. We applied statistical tests to the log response ratio, $L$, which is less sensitive than the response ratio to errors in estimating the denominator of a ratio (Hedges et al. 1999), but figures show means and confidence limits for the response ratio itself, which are obtained by exponentiating $L$ and its confidence limits. We also computed Hedges' $d$ for each agent in the usual fashion, including the correction for small sample size, $J$ (Gurevitch and Hedges 2001); negative values of $d$ indicate that the agent reduced plant performance.

In factorial experiments, the effect of an agent can be measured in two ways: by comparing the treatments with and without that agent in the absence of the other agent or by comparing the mean performance in the two treatments in which the agent is present vs. the two treatments in which the agent is absent (analogous to a main effect in a two-way ANOVA). We refer to these two measures as the "individual" vs. "overall" effects of an agent. The individual effect is more comparable to impact measures from studies of enemies and mutualists in isolation, whereas the overall effect provides a more realistic measure of an agent's effect across levels of the other species. Gurevitch et al. (2000) developed measures of individual, overall, and interaction effects for a $2 \times 2$ factorial meta-analysis of the effects of competition and predation; we modified their measures for our analyses of enemy and mutualist effects (Appendix B). In particular, we designed our interaction effect such that, using both $L$ and $d$, a positive value indicates that the interaction between the two agents tends to enhance plant performance (i.e., performance is higher in the combined treatment than the sum of the individual effects of the two agents would predict).

To compute the mean effect size across a group of studies, we computed the weighted mean of the log response ratios from the individual studies, where the weights are the inverses of the sampling variances of the effect sizes in each study. We test for differences in mean effect size among groups (e.g., herbivores vs. pathogens, aboveground vs. belowground herbivores, etc.) using a random effects model, which allows for the possibility that the true effect sizes may vary among studies within a group (Gurevitch and Hedges 2001). For effect sizes using $d$, the sampling variances were computed as described in the appendix of Gurevitch et al. (2000); for effect sizes using $L$, sampling variances are given in Appendix B. To compare mean effect sizes among 


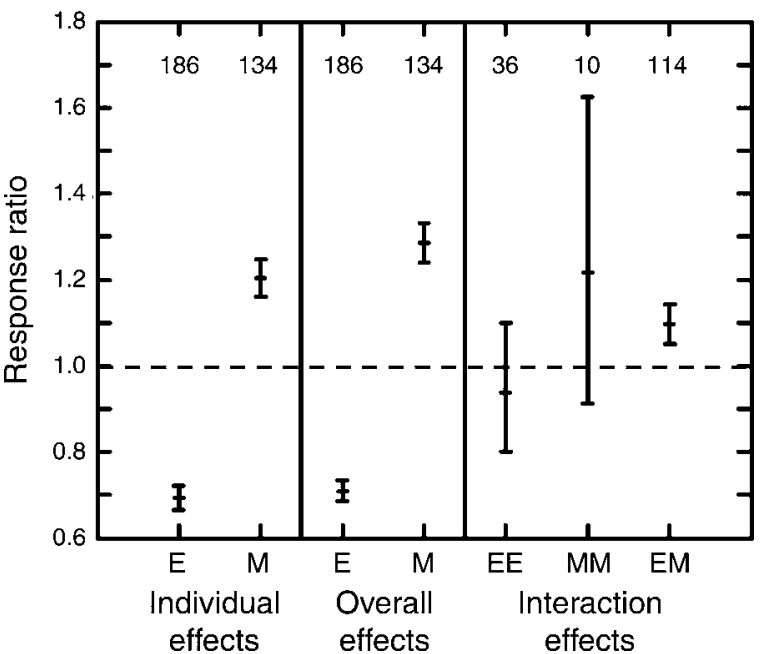

FIG. 1. Individual, overall, and interaction effects (mean and $95 \% \mathrm{CI}$ ) of enemies (E) and mutualists (M) on plant performance from the full data set (see Methods: Calculation and comparison of effect sizes for details). Individual effects measure an agent's influence in isolation, and overall effects measure its influence across levels of another agent. For individual and overall effects, a response ratio of 1 indicates that the agent had no effect on the plant, while ratios $>1$ and $<1$ show a proportional increase and decrease, respectively, in plant performance in the presence of the agent. For the interaction effect between two agents, the response ratio equals 1 if the effects of the two agents are independent and is $>1$ or $<1$ if plant performance is, respectively, greater than or less than the product of the individual effects of the two agents. Sample sizes are indicated at the top.

groups (using a mixed model; Gurevitch and Hedges 2001: Eq. 18.21), we performed homogeneity tests in which the (weighted) among-group sum of squares $Q_{\mathrm{b}}$ was compared to the critical value $(\alpha=0.05)$ of the chisquare distribution with degrees of freedom equal to the number of groups minus 1. Because positive and negative effects on plant performance scale differently using the $\log$ response ratio, we used a procedure described in Appendix B (which involves bootstrapping the mean $\log$ ratio effect sizes and back-transforming them) to compare the mean magnitudes of enemy and mutualist effects.

\section{RESULTS}

The full data set incorporated information from 68 articles and included 36 enemy-enemy studies, 10 mutualist-mutualist studies, and 114 enemy-mutualist studies, while the most conservative data set (requiring both different agent pairs and different controls) comprised 27, 7, and 45 studies, respectively (Appendix A). We present graphical results from the full data set using the response ratio and note whether those results also hold for the reduced data sets and for Hedges' $d$. Results using Hedges' $d$ are presented in detail in Appendix C.
As expected, enemies reduced and mutualists increased plant performance (Fig. 1, Appendix C). This was true for both individual and overall effects and for random draws from all three reduced data sets (Appendix E). Nonetheless, there was considerable heterogeneity in effect sizes within groups (i.e., $Q$ tests were significant $[P<0.001]$ for all effects in Fig. 1). Some agents traditionally viewed as "enemies" increased plant performance, and some "mutualists" decreased plant performance. Comparing enemies and mutualists, the magnitude of the mean individual effect of enemies on plant performance (indicated by the distance away from a response ratio of 1 in Fig. 1) was significantly greater $(P=0.027)$ than the magnitude of the mean individual effect of mutualists, although the magnitudes of the overall effects did not differ significantly $(P=$ 0.369; Appendix B).

Enemies did not, on average, influence the effects on plant performance of other enemies. Likewise, mutualists did not, on average, show nonindependent effects on plants (Fig. 1, Appendix C). Of the 5000 random draws from the data using different controls but allowing agent pairs to be repeated, $<10 \%$ showed a mean interaction between enemies or between mutualists that led to increased plant performance (Appendix E). This confirms that, on average, effects of pairs of enemies and pairs of mutualists were independent. However, this average masks significant interactions between particular pairs of agents, some of which enhanced and others of which decreased plant performance. Nearly one-third of enemy-enemy and mutualist-mutualist studies combined detected significant interaction effects (Fig. 2).

When enemies and mutualists were both present, they did interact such that plant performance was better than would be expected from an independent model (Fig. 1). The analysis of Hedges' $d$ also showed a positive enemymutualist interactive effect on average, but the 95\% CI overlaps zero (Appendix C). The reduced data sets supported this result, with $>80 \%$ of the 5000 random draws showing a positive interaction (Appendix E). When comparing subgroups for which we have sufficient data, the interaction remains significantly positive for pathogens paired with bacterial mutualists (mean [95\% CL] of $L, 0.10$ [0.06, 0.15]; $n=28)$ and for pathogens with arbuscular mycorrhizal (AM) fungi (0.14 [0.05, $0.24] ; n=33)$, but not for herbivores with AM fungi (0.4 $[-0.03,0.11] ; n=38)$ or herbivores with pollinators $(0.4$ $[-0.5,0.54] ; n=13)$. Despite these apparent differences, among-group heterogeneity in the enemy-mutualist interaction effect was not significant $\left(Q_{\mathrm{b}}=1.27\right.$, df $=$ $3, P=0.74)$.

Individual classes of enemies and mutualists differed in their effects on plant performance (Fig. 3). Pathogens were significantly more detrimental to plant performance than were herbivores. One possible explanation for this pathogen/herbivore difference is that pathogen effects were significantly greater in the greenhouse than in the field (Fig. 4a, Appendix E), and 65 of 84 pathogen 


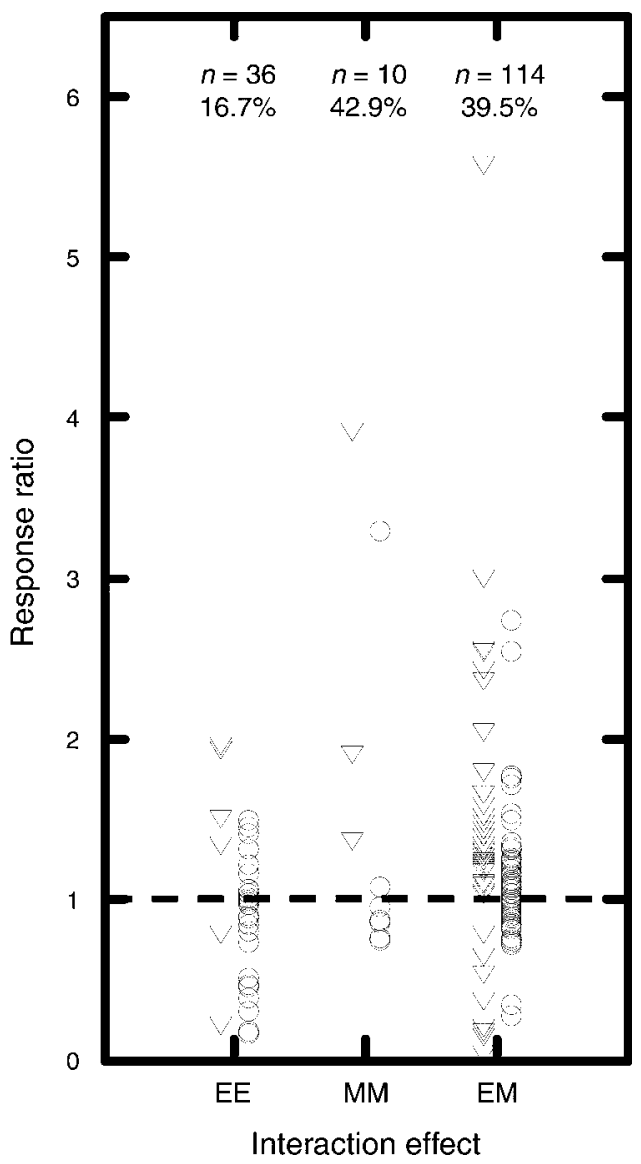

FIG. 2. Interaction effect sizes (E, enemies; $M$, mutualists) in individual studies from the full data set; significant $(P<0.05)$ effects are indicated by inverted triangles, nonsignificant effects by circles. Sample sizes and percentages of studies with significant interaction effects are indicated at the top. See Fig. 1 for an explanation of the response ratio.

studies in our data set were performed in the greenhouse (compared to 47 of 100 herbivore studies). Thus, the observed greater effect of pathogens could result simply from their being more frequently studied in the greenhouse environment. We therefore analyzed separately greenhouse and field experiments. In the greenhouse, the effect of pathogens (mean [95\% CL] of $L$, $-0.46[-0.52,-0.40])$ was still significantly stronger $\left(Q_{\mathrm{b}}\right.$ $=11.29, P<0.001)$ than the effect of herbivores $(-0.25$ $[-0.31,-0.19])$. This difference was not observed in the field (pathogen effect, $-0.27[-0.41,-0.12]$; herbivore effect, $-0.33[-0.45,-0.21] ; Q_{\mathrm{b}}=0.34, P=0.56$ ), perhaps because we were able to uncover only 19 cases of pathogens used in factorial field experiments. There was also significant variation among the classes of mutualists, with pollinators and mycorrhizal fungi benefiting plants more than soil-dwelling mutualistic bacteria (Fig. 3).

Mutualist effects, enemy-enemy interactions, and enemy-mutualist interactions were consistent when compared in field vs. greenhouse, on herbaceous vs. woody plants, and on crop vs. non-crop plants (Fig. 4, Appendix E; there were too few data to evaluate how mutualist-mutualist interactions differed between these groups). Pathogens were more detrimental to herbaceous than to woody plants (Fig. 4b). This could result from the fact that the percentage of pathogen studies conducted in the greenhouse was higher for herbaceous (81.5\%) than for woody (63.2\%) plants and pathogens were more damaging in the greenhouse than in the field (Fig. 4a). Thus we again separately analyzed greenhouse and field experiments. In the greenhouse, pathogens did have a significantly more negative effect on herbaceous than on woody plants $(L=-0.50[-0.58,-0.42]$ and $-0.25[-0.31,-0.19]$, respectively; $\left.Q_{\mathrm{b}}=8.96, P=0.003\right)$. This difference was not significant in the field (herbaceous plants, -0.27 [ $-0.45,-0.09]$; woody plants, -0.21 [-0.35, -0.07$\left.] ; Q_{\mathrm{b}}=0.01, P=0.924\right)$, but again, sample sizes were much smaller in the field. Herbivores had more detrimental effects on crop than on non-crop plants (Fig. 4c), but proportionally more herbivore studies were conducted in the greenhouse for crops $(80.6 \%)$ than for non-crops $(28.3 \%)$. Once again, the stronger herbivore effects on crop plants held up in the greenhouse (crops, -0.307 [ $-0.379,-0.235]$; non-crops, $\left.-0.143[-0.305,0.020] ; Q_{\mathrm{b}}=10.10, P=0.002\right)$ but not in the field (crops, $-0.393[-0.760,-0.026]$; non-crops, -0.350 [-0.499, -0.201$\left.] ; Q_{\mathrm{b}}=0.06, P=0.815\right)$.

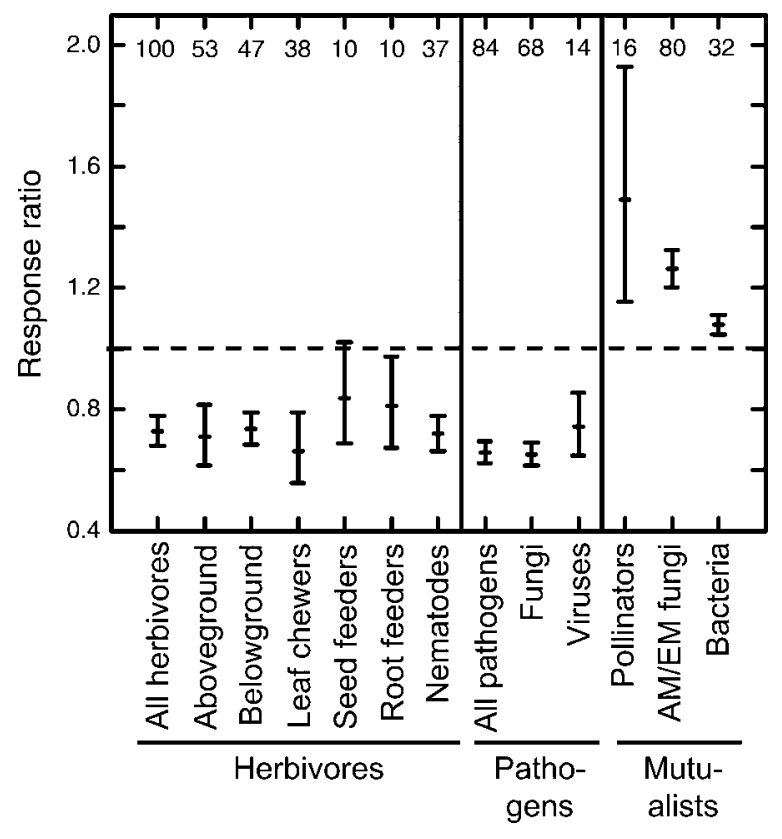

FIG. 3. Individual effects (mean and 95\% CI) of different classes of enemies and mutualists from the full data set. Sample sizes are indicated at top. Homogeneity tests: herbivores vs. pathogens, $Q_{\mathrm{b}}=5.54, P=0.019$; above- vs. belowground herbivores, $Q_{\mathrm{b}}=0.06, P=0.814$; four classes of herbivores, $Q_{\mathrm{b}}$ $=2.76, P=0.429$; fungal vs. viral pathogens, $Q_{\mathrm{b}}=2.34, P=$ 0.126; three classes of mutualists (AM, arbuscular mycorrhizal; EM, ectomycorrhizal), $Q_{\mathrm{b}}=12.82, P=0.002(\mathrm{df}=$ number of classes minus 1). 

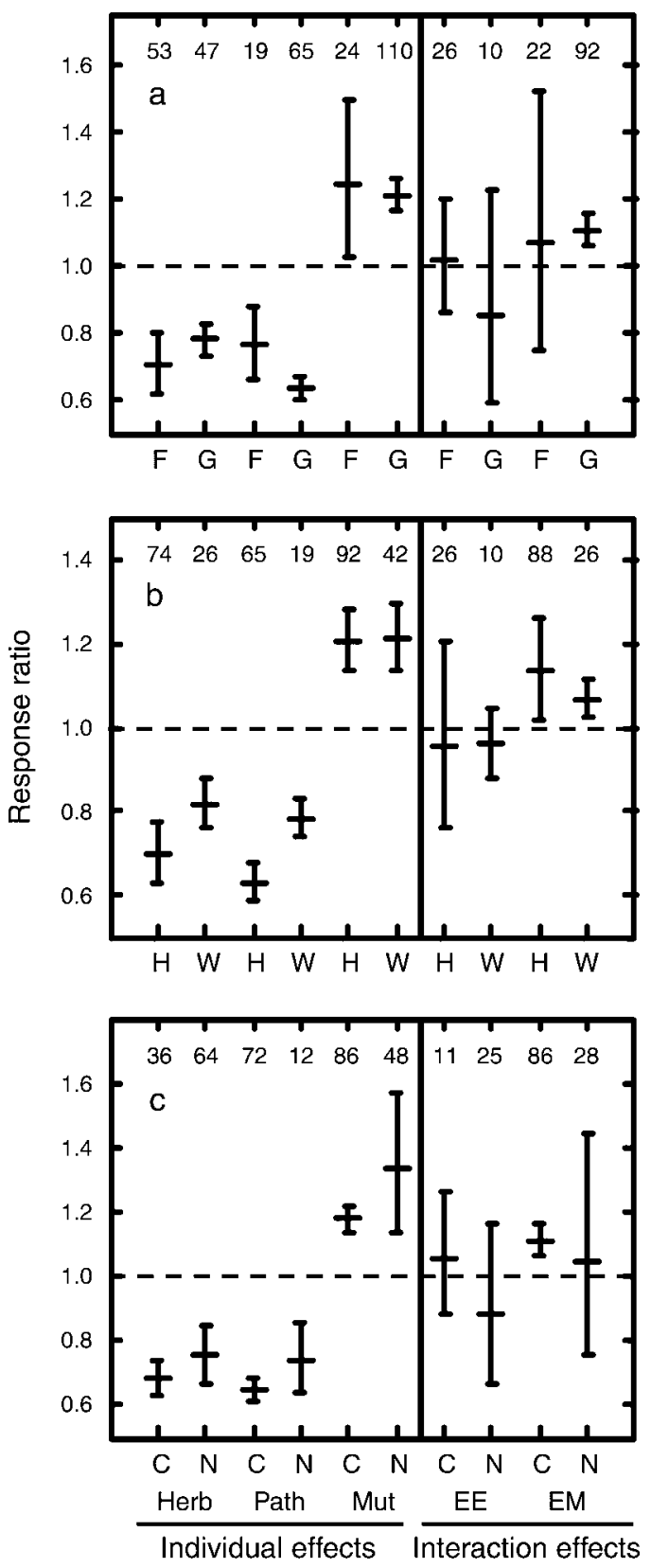

FIG. 4. Influence of environment (mean and 95\% CI) on individual (herbivore [Herb], pathogen [Path], and mutualist [Mut]) and interaction (enemies [E] and mutualists [M]) effects from the full data set for (a) field (F) vs. greenhouse (G), (b) plant life form (herbaceous $[\mathrm{H}]$ vs. woody [W]), and (c) agronomic status (crop [C] vs. non-crop $[\mathrm{N}]$ ). Sample sizes are indicated at top.

When enemies and mutualists were both present, plants benefited from the interaction effect in greenhouse studies (lower 95\% CL > 1), but not in field studies (Fig. 4a). Likewise, the interaction effect benefited crop plants but not non-crop plants (Fig. 4c). However, the differences in the enemy-mutualist interaction effect between greenhouse and field and between crop and non-crop studies were not statistically significant (Appendix E). The type of enemy and mutualist probably strongly influenced the fact that the enemy-mutualist interaction effect was significant only in greenhouse studies and in crop species. In the 92 enemy-mutualist greenhouse studies, the mutualists were either mycorrhizal fungi $(n=64)$ or bacteria $(n=$ $28)$, and the enemies were mostly pathogenic fungi ( $n=$ 57) and nematodes $(n=27)$, with a few pathogenic bacteria $(n=2)$ and insects $(n=6)$. In contrast, the mutualists in the 22 field studies were either pollinators $(n=15)$ or mycorrhizal fungi $(n=7)$, and the enemies included only a few nematodes $(n=3)$ and pathogenic fungi $(n=2)$ but a broad mix of other taxa (including nectar robbers and vertebrate and invertebrate herbivores). Similarly, the 86 enemy-mutualist studies on crops included 57 studies of AM fungi, 28 studies of mutualistic bacteria, and one pollinator study, while half of the 28 non-crop studies were pollinator studies and half were mycorrhizal fungal studies.

The type of performance measure (size vs. reproduction) did not influence the patterns in the results except in the case of the overall effects of mutualists, in which the benefit was greater in studies using reproductive measures (generally pollination studies) than in studies using size measures (generally microbial mutualists; Appendix D).

\section{Discussion}

\section{Magnitude of enemy vs. mutualist effects}

In isolation from other agents, enemies caused a proportional reduction in plant performance that was significantly greater in magnitude than the proportional increase in performance caused by mutualists. In contrast, the mean magnitudes of positive and negative effects across all levels of the other interacting agents (i.e., the overall effects) were comparable. The difference between individual and overall mutualist effects is linked to the result that enemy-mutualist interaction effects are on average positive (Fig. 1); overall effects of mutualists (at least those in enemy-mutualist studies, which are far more numerous than mutualist-mutualist studies in our database) include the influence of this interaction effect whereas individual effects of mutualists do not. This result suggests that, to accurately weigh the impact a particular type of agent has on plant fitness, it may be important to measure that impact in the presence of other biotic interactions in which the plant engages simultaneously. Indeed, many mutualistic interactions may be inherently indirect (e.g., "protectors" such as ant defenders or mutualistic bacteria that compete with pathogens may benefit plants only when the plants' enemies are present; Bronstein and Barbosa 2002). The similar magnitudes of enemy and mutualist effects suggest that in multispecies communities their impacts on plant performance may often cancel one another out and that both enemies and mutualists need to be 
considered when weighing the impact of biotic interactions on the primary productivity of ecosystems.

\section{Variation among classes of enemies and plant groups}

The herbivore effects in our studies were somewhat stronger on average, when measured using Hedges' $d$ (mean [95\% CL], -0.67 [-0.83, -0.51]; Appendix C), than the mean observed in Bigger's and Marvier's (1998) meta-analysis $(-0.47[-0.59,-0.35])$. A likely explanation for the difference is that Bigger and Marvier found vertebrate herbivores to have weaker effects than invertebrate herbivores and their data set included more vertebrate than invertebrate studies (whereas only $14 \%$ of the herbivory studies in our data set involved vertebrates, likely because fewer factorial experiments have been conducted with vertebrates). Although Bigger and Marvier excluded greenhouse studies, we found that herbivore effects were, if anything, weaker in the greenhouse than in the field (Fig. 4a).

Although based chiefly on greenhouse experiments, the data suggest that pathogens have a greater effect on plants than do herbivores. If this pattern holds up in the field with larger sample sizes, then three intriguing predictions arise: (1) pathogens may exert stronger selection on resistance traits, particularly those of herbaceous plants, than do herbivores; (2) escape from their native pathogens may be a more important factor in the success of invasive plants (through "enemy release"; Torchin and Mitchell 2004) than escape from native herbivores; and (3) use of pathogens may hold more promise for biocontrol of invasive plants than use of herbivores.

Even though herbivore effects were weaker on average than pathogen effects, herbivores did have a significantly greater impact on crop than on non-crop plants (at least in the greenhouse), while pathogen effects did not differ significantly between the two (although the trend was in the same direction; Fig. 4c, Appendix E). Should this result hold up (particularly with more field studies of pathogen effects on non-crops), it may indicate that, upon domestication, plants may lose some of their resistance or tolerance to herbivores. Alternatively, herbivores that consume crop plants may have evolved more detrimental ways of exploiting their hosts.

\section{Magnitude and direction of interaction effects}

On average, there was no tendency for the interaction between enemies or between mutualists to either increase or decrease plant performance, yet many individual studies deviate from the average, showing significant positive (in both enemy-enemy and mutualist-mutualist studies) or significant negative (in enemy-enemy studies) interaction effects (Fig. 2). Thus it may be difficult to predict a priori whether any particular enemy-enemy or mutualist-mutualist pair will have synergistic or antagonistic effects on plant performance. As more factorial studies accumulate, we may be able to identify taxonomic or other features of plants, enemies, and mutualists that influence the direction of the interaction effects. Hougen-Eitzman and Rausher (1994; also see Iwao and Rausher 1997, Stinchcombe and Rausher 2001) have argued that co-evolution between plants and interacting species will be diffuse if the pattern of natural selection imposed by one species on plant traits is altered in the presence of a second species. Antagonism or synergism between the effects of separate agents on mean plant performance may or may not reflect changes in the pattern of selection (Inouye and Stinchcombe 2001, Strauss et al. 2005). Although the divergent directions of enemy-mutualist interaction effects may render coevolutionary responses difficult to anticipate, the abundance of interactive effects in the database suggests the widespread potential for diffuse coevolutionary interactions between plants, their enemies, and their mutualists.

In our central analysis, the presence of mutualists mitigated the negative effects of enemies on plant performance. When the enemy-mutualist studies were divided into categories, the significantly positive interaction effect remained in interactions between pathogenic and mutualistic soil bacteria and between pathogenic and mutualistic soil fungi, both of which are to be expected a priori (Borowicz 2001). Borowicz (2001), in a meta-analysis using Hedges' $d$ and including many of the same studies in our database, also found that on average the interactive effect of fungal pathogens and mycorrhizal fungi was positive. In contrast to our study, Borowicz (2001) found that the interaction between nematodes (which we have classified with other herbivores in our analysis) and mycorrhizal fungi had a negative impact on plant growth. This difference suggests that interactive effects involving one type of herbivore may not carry over to a broader array of herbivore types, a suggestion that must be tempered by the fact that most of the bacterial and fungal studies took place in the greenhouse, whereas the (fewer) studies of herbivores or nectar robbers combined with pollinators were performed entirely in the field.

Our results have implications for understanding and controlling invasions of exotic plants. Given that enemies and mutualists tend to have positive interaction effects, if both herbivores and mutualists are lost when a plant is introduced to a new locale, its performance may be less than would be predicted by summing the effects of the individual losses (even if performance is on the whole better in the introduced than in the native range). Regarding control of exotic invasions, because the average enemy-enemy interaction effects are near zero, an across-the-board recommendation against multiple introductions to avoid antagonistic interactions between biocontrol agents is not justified by the data (nor is the alternative recommendation that synergistic effects will always favor multiple introductions). Instead, the existence of significant positive and negative interaction effects in individual studies means that release of multiple agents needs to be decided on a case-by-case 
basis, weighing any potential benefits of synergism against other risks inherent in introducing nonnative species (McEvoy and Coombs 1999).

\section{Caveats and suggestions for future work}

The results of any meta-analysis are conditional on the set of studies included. Because we sought to address questions about interaction effects, we included only factorial experiments in our database. Inclusion of the large number of existing single-agent studies, many of which have been included in previous meta-analyses, might alter our conclusions about the size of direct effects of enemies or mutualists. However, our finding that the magnitude of mutualist effects differs when computed as individual vs. overall effects suggests that single-agent studies may not always capture the true effect an agent has in a more diverse community.

This meta-analysis suggests the need for future experiments of certain kinds. Our database was notably depauperate in factorial studies involving two mutualists, limiting our power to detect interactions between them. With growing recognition (e.g., Stanton 2003) that many plant species engage in multiple, simultaneous mutualisms, more studies of mutualist-mutualist interactions may soon appear. Moreover, several groupings of agent and plant types have never or rarely been manipulated factorially in the field. As ecologists' ultimate goal is to understand agent effects in the field, our conclusions should be revisited as more field studies become available. In particular, as difficult as they may be to perform, more field studies involving belowground enemies and mutualists and pairs of belowground mutualists are sorely needed.

Several effect size differences (e.g., the greater effect of pathogens than herbivores) were significant in the greenhouse but not the field. One possible explanation is that the greenhouse environment created unrealistic effects. But a second possibility is that effects were less accurately measured in the field, masking real differences seen in the greenhouse. Among studies in our database, within-treatment sample sizes averaged three times higher in the field than in the greenhouse (medians, 18 vs. 6 in field and greenhouse, respectively; rank sum test, $P<0.001$ ), but the ratio of the within-treatment standard errors to the within-treatment means of plant performance was significantly higher on average in the field $(0.36)$ than in the greenhouse $(0.28$; rank sum test, $P<0.001)$. This result implies that, despite greater experimental effort, effect sizes were nonetheless measured less accurately in the field, perhaps due to microenvironmental variation, greater genetic variation among replicate plants, or uncontrolled variation in the densities of additional interacting species. Thus in addition to the need for a greater number of field studies, our results point to the need for even larger field studies if the accuracy of effect size estimates is to be improved.
Finally, we note that different types of study tend to use different plant performance measures, often for good biological reasons (e.g., fungal mutualists directly affect plant growth, and pollinators affect reproductive output). However, individual growth and reproduction rarely contribute equally to fitness or population growth. The ideal approach would be to integrate effects of agents on different aspects of plant performance into a single measure, such as a population growth rate, as was done by Garcia and Ehrlén (2002) and Knight (2004). Such an approach requires that all the plant's demographic rates (survival, growth, and reproduction) be measured, even those that are not affected by the agents. This additional work would not only allow more direct comparison of enemy and mutualist effects, it would also provide the means to extrapolate the consequences of agents' impacts on individual plant performance to the dynamics of plant populations.

\section{ACKNOWLEDGMENTS}

Author order after the second is alphabetical. This work was conducted as part of the Biotic Interactions Working Group supported by the National Center for Ecological Analysis and Synthesis, a Center funded by NSF (Grant \#DEB-0072909), the University of California, and the Santa Barbara campus.

\section{Literature Cited}

Bigger, D. S., and M. A. Marvier. 1998. How different would a world without herbivory be? A search for generality in ecology. Integrative Biology 1:60-67.

Borowicz, V. A. 2001. Do arbuscular mycorrhizal fungi alter plant-pathogen relations? Ecology 82:3057-3068.

Bronstein, J. L., and P. Barbosa. 2002. Multi-trophic/multispecies mutualistic interactions: the role of non-mutualists in shaping and mediating mutualisms. Pages 44-65 in B. Hawkins and T. Tscharntke, editors. Multitrophic level interactions. Cambridge University Press, Cambridge, UK.

Denoth, M., L. Frid, and J. H. Myers. 2002. Multiple agents in biological control: Improving the odds? Biological Control $24: 20-30$

Garcia, M. B., and J. Ehrlén. 2002. Reproductive effort and herbivory timing in a perennial herb: fitness components at the individual and population levels. American Journal of Botany 89:1295-1302.

Goldberg, D. E., T. Rajaniemi, J. Gurevitch, and A. StewartOaten. 1999. Empirical approaches to quantifying interaction intensity: competition and facilitation along productivity gradients. Ecology 80:1118-1131.

Gomez-Aparicio, L., R. Zamora, J. M. Gomez, J. A. Hodar, J. Castro, and E. Baraza. 2004. Applying plant facilitation to forest restoration: a meta-analysis of the use of shrubs as nurse plants. Ecological Applications 14:1128-1138.

Gurevitch, J., and L. V. Hedges. 2001. Meta-analysis: combining the results of independent experiments. Pages 347-369 in S. M. Scheiner and J. Gurevitch, editors. Design and analysis of ecological experiments. Second edition. Oxford University Press, Oxford, UK.

Gurevitch, J., J. A. Morrison, and L. V. Hedges. 2000. The interaction between competition and predation: a metaanalysis of field experiments. American Naturalist 155:435453.

Gurevitch, J., L. L. Morrow, A. Wallace, and J. S. Walsh. 1992. A meta-analysis of competition in field experiments. American Naturalist 140:539-572.

Hamback, P. A., and A. P. Beckerman. 2003. Herbivory and plant resource competition: a review of two interacting interactions. Oikos 101:26-37. 
Hawkes, C. V., and J. J. Sullivan. 2001. The impact of herbivory on plants in different resource conditions: a metaanalysis. Ecology 82:2045-2058.

Hedges, L. V., J. Gurevitch, and P. S. Curtis. 1999. The metaanalysis of response ratios in experimental ecology. Ecology 80:1150-1156.

Hedges, L. V., and I. Olkin. 1985. Statistical methods for metaanalysis. Academic Press, Boston, Massachusetts, USA.

Hougen-Eitzman, D., and M. D. Rausher. 1994. Interactions between herbivorous insects and plant-insect coevolution. American Naturalist 143:677-697.

Inouye, B., and J. R. Stinchcombe. 2001. Relationships between ecological interaction modifications and diffuse coevolution: similarities, differences, and causal links. Oikos 95:353-360.

Irwin, R. E., A. K. Brody, and N. M. Waser. 2001. The impact of floral larceny on individuals, populations, and communities. Oecologia 129:161-168.

Iwao, K., and M. D. Rausher. 1997. Evolution of plant resistance to multiple herbivores: quantifying diffuse coevolution. American Naturalist 149:316-335.

Knight, T. M. 2004. The effect of herbivory and pollen limitation on a declining population of Trillium grandiflorum. Ecological Applications 14:915-928.

Lortie, C. J., and R. M. Callaway. 2006. Re-analysis of metaanalysis: support for the stress-gradient hypothesis. Journal of Ecology 94:7-16.

Maestre, F. T., F. Valladares, and J. F. Reynolds. 2005. Is the change of plant-plant interactions with abiotic stress predictable? A meta-analysis of field results in arid environments. Journal of Ecology 93:748-757.

Maestre, F. T., F. Valladares, and J. F. Reynolds. 2006. The stress-gradient hypothesis does not fit all relationships between plant-plant interactions and abiotic stress: further insights from arid environments. Journal of Ecology 94:1722.

McEvoy, P. B., and E. M. Coombs. 1999. Biological control of plant invaders: regional patterns, field experiments, and structured population models. Ecological Applications 9: 387-401.

Neill, W. 1974. The community matrix and interdependence of the competition coefficients. American Naturalist 108:399408.

Rosenberg, M. S., K. A. Garrett, Z. Su, and R. L. Bowden. 2004. Meta-analysis in plant pathology: synthesizing research results. Phytopathology 94:1013-1017.

Sih, A., G. Englund, and D. Wooster. 1998. Emergent impacts of multiple predators on prey. Trends in Ecology and Evolution 13:350-355.

Stanton, M. L. 2003. Interacting guilds: moving beyond the pairwise perspective on mutualisms. American Naturalist 162:S10-S23.

Stinchcombe, J. R., and M. D. Rausher. 2001. Diffuse selection on resistance to deer herbivory in the ivyleaf morning glory, Ipomoea hederacea. American Naturalist 158:376-388.

Strauss, S. Y., and R. E. Irwin. 2004. Ecological and evolutionary consequences of multispecies plant-animal interactions. Annual Review of Ecology Evolution and Systematics 35:435-466.

Strauss, S. Y., H. Sahli, and J. K. Conner. 2005. Toward a more trait-centered approach to diffuse (co)evolution. New Phytologist 165:81-89.

Torchin, M. E., and C. E. Mitchell. 2004. Parasites, pathogens, and invasions by plants and animals. Frontiers in Ecology and the Environment 2:183-190.

Wilbur, H. M. 1972. Competition, predation, and the structure of the Ambystoma-Rana sylvatica community. Ecology 53:321.

Wootton, J. T. 1993. Indirect effects and habitat use in an intertidal community - interaction chains and interaction modifications. American Naturalist 141:71-89.

Yeo, J. J. 2005. Effects of grazing exclusion on rangeland vegetation and soils, East Central Idaho. Western North American Naturalist 65:91-102.

\section{APPENDIX A}

Articles and data used in the meta-analysis (Ecological Archives E088-063-A1).

\section{APPENDIX B}

Measures used for individual, overall, and interaction effect sizes and their sampling variances (Ecological Archives E088-063A2).

\section{APPENDIX C}

Tables showing effect sizes using Hedges' $d$ (Ecological Archives E088-063-A3).

\section{APPENDIX D}

A figure showing effect sizes using different measures of plant performance (Ecological Archives E088-063-A4).

\section{APPENDIX E}

Tables showing results from subsampling studies from the entire data set (Ecological Archives E088-063-A5).

\section{SUPPLEMENT 1}

Information on studies used in the meta-analysis (Ecological Archives E088-063-S1).

\section{SUPPLEMENT 2}

MATLAB code used to perform factorial meta-analyses using Hedges' $d$ and the log response ratio (Ecological Archives E088063-S2). 